\section{DEFORMATION OF EARTH'S SURFACE CAUSED LOADING OF TALL BUILDING}

\section{ABSTRACT}

Tall buildings can cause deformations of the earth's crust for long distances from the area of their realization. Monitoring the deformation of tall big buildings from geodetic networks realised near the building can also have deformations from this point of view, and analysis of the measurements can result in false conclusions. The loading effect of the earth crust must be excluded from the measurement parameters. The paper presents a model computation of the loading effect produced by tall buildings constructed of simple geometric forms on the earth's crust.

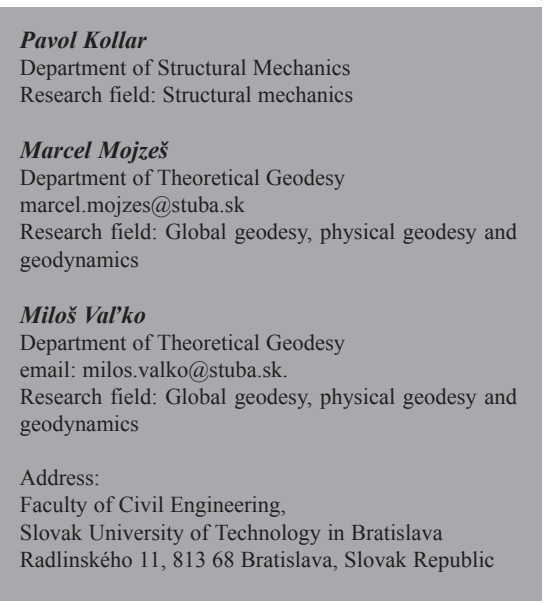

\section{KEY WORDS}

- Crust deformation,

- elasticity,

- viscosity,

- viscoelasticity

\section{INTRODUCTION}

When studying of surface loading of the earth, we can consider two extreme cases of deformation: absolutely rigid with no deformable body or a fluid body without any resistance form external pressure. The actual response of loading on the Earth's surface is between these extreme cases. The materials of the Earth's surface can have common properties, i.e., elastic, viscoelastic or plastic. If the external loading is only for a time short, then the deformation is given only consists elastic properties. This means that when the external forces are removed the Earth's surface regain its original undeformed shape. An ideal elastic material lacks memory, i.e., any stress or deformation is uniquely determined by the final values of the loading. On the other hand, if the force effect is long term, then elastic and rheological effects can be found in the reaction of the material to external loading. Those effects are modelled as viscoelastic and plastic. In both cases is there a new factor - time. The time factor directly connects with the mechanical memory of the material depending on the long-term effect and history of the loading process. In contrast to elastic deformation, viscous and plastic deformations are not reversible, i.e., after by removing the loading, the new shape of the Earth's surface is not the same as its original undeformed shape. Those effects are not viscoelastic but plastic.

A deeper knowledge of the physical - mechanical properties of materials and modern computer equipment can allow for a very precise model of the reaction of the Earth's surface to loading over time, which is the next step in understanding complicated phenomena of the Earth's crust. Geodesists need to know these laws of surface deformation and their time evolution for of geodetic networks the design, analysis of repeat measurements or time series of measured parameters.

This paper is analyses the surface deformation of the Earth caused by symmetric loading of its boundaries. We consider that the background is an elastic body modelled as a homogenous and isotropic half-space without any time response. The results in analytic form are presented using actual examples. 


\section{DEFORMATION OF A BACKGROUND BY AXISYMETRIC LOADING}

This paper shows how to determine deformations of the Earth's surface by axisymmetric loading of its boundaries (i.e. plane $z=0$ ). The solution is based on these conditions:

- The basement is a homogeneous, isotropic and perfectly elastic body (half-space) of the Boussinesq type,

- The material properties of the elastic body are: Young's modulus of elasticity $E_{\mathrm{z}}$ (unit MPa) and Poisson ratio $\mu_{\mathrm{z}}$ (dimensionless),

- The boundary of the half-space (surface $z=0$ ) is on the area of a circle with a radius $R$ (unit $\mathrm{m}$ ) (Fig. 1a) or on an annulus area $\mathrm{r}_{1} \leq \mathrm{r} \leq \mathrm{r}_{2}$ loaded by a permanent, uniform, and normal loading of a magnitude $\mathrm{p}_{\mathrm{i}}(i=1,2)$ (unit MPa) (Fig. 1b).
The construction of a half-space boundary flexure surface $v_{\mathrm{z}}(\rho)$, where $\rho=r / R$, by loading $p_{1}$ of its boundaries is known as the first boundary problem in the theory of elasticity. In this paper we present the Green functions approach. First, we have to consider two auxiliary problems: in the first problem we try to find the function for the deformation $v_{z}^{*}(\rho, \bar{\rho})$ half-space boundary by unit loading, which is uniformly distributed on a circular line with a radius $\bar{\rho}$ (Fig. 3). In the second problem we determine the integral relations for the displacements $v_{\mathrm{z}}(\rho)$, which are caused by the continuous loading given in Figs. 1a, $1 \mathrm{~b}$.

The vertical displacement at $B(r, \varphi)$ on the half-space boundary $(z$ $=0$ ) by the unit force $P=1$ located at $A(\bar{r}, \psi)$ (Fig. 2) is given by Boussinesq's formula (Boussinesq, 1885)

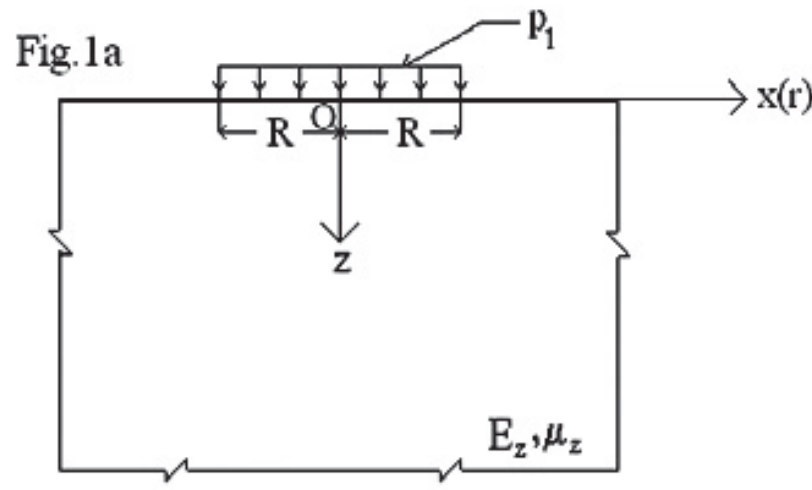

Fig. $1 b$
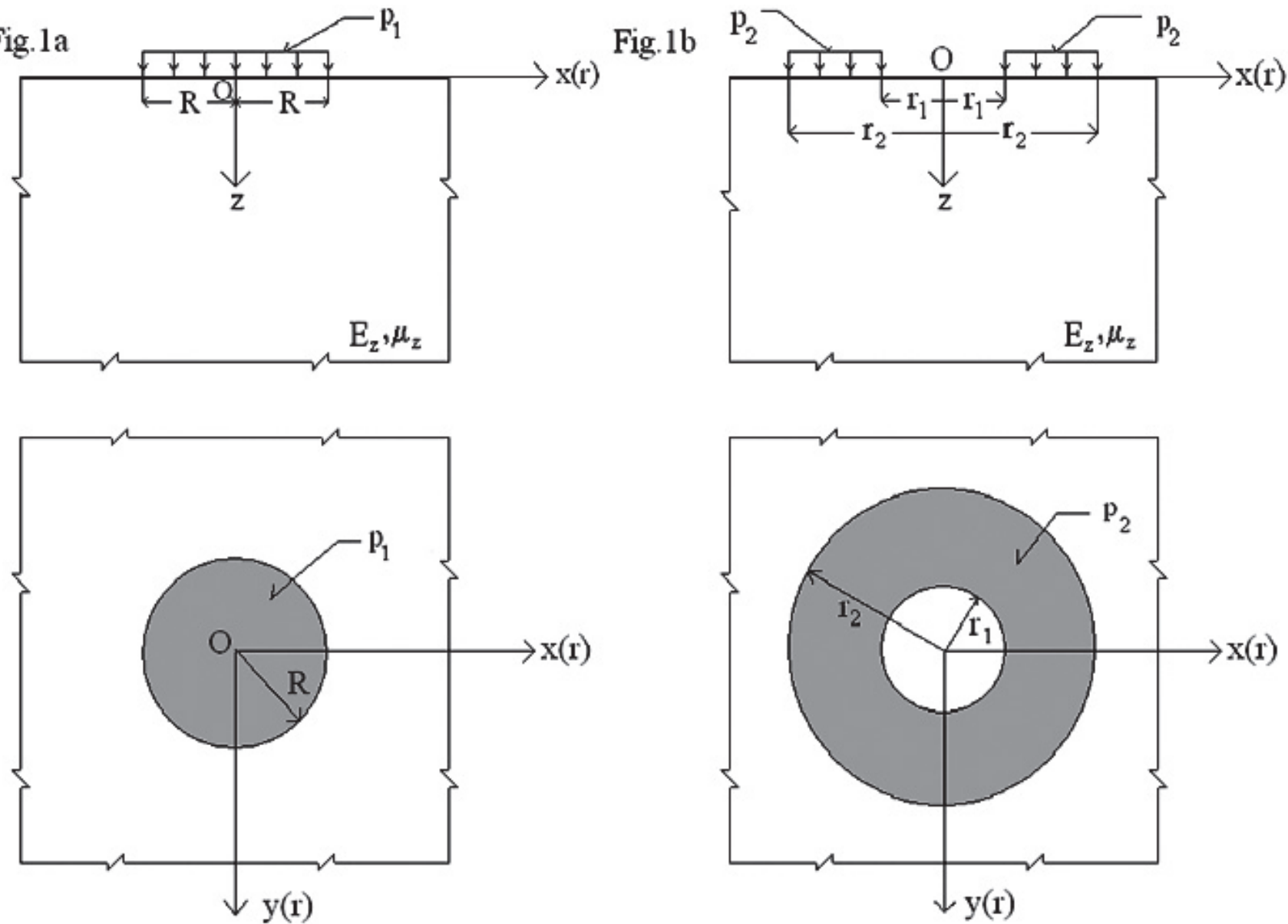

Fig. 1 Schema of loading a half-space boundary $(z=0)$ on the area of a circle with the radius $R$ (Fig. 1a) and an annular area $r_{1} \leq r \leq r_{2}$ (Fig. 1b) 


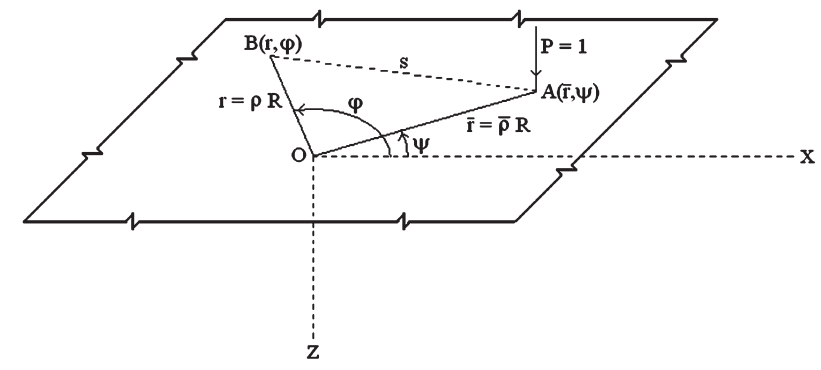

Fig. 2 Geometric schema of half-space loading by concentrated force $P=1$

$$
\bar{v}_{z}(\rho, \bar{\rho}, \varphi, \psi)=\frac{1-\mu_{z}^{2}}{\pi E_{z}} \frac{1}{s}
$$

where

$s=R \sqrt{\rho^{2}+\bar{\rho}^{2}-2 \rho \bar{\rho} \cos (\varphi-\psi)}, \quad \rho=\frac{r}{R}, \quad \bar{\rho}=\frac{\bar{r}}{R}$.

Now change the unit loading $P=1$ by the elementary loaded $d P=1 R \bar{\rho} d \psi$, caused by circular line part $R \bar{\rho} d \psi$ (Fig. 3). With the integration of (1) for the whole circumference of the circular line, the vertical displacement of $R(\rho R, \varphi)$, which is caused by unit's uniformly loaded caused by circular line of radius $\bar{r}$, we can write in the form

$v_{z}^{*}(\rho, \bar{\rho})=\frac{\left(1-\mu_{z}^{2}\right)}{\pi E_{z}} \int_{0}^{2 \pi} \frac{\bar{\rho} d \psi}{\sqrt{\rho^{2}+\bar{\rho}^{2}-2 \rho \bar{\rho} \cos (\varphi-\psi)}}$.

In respect of problem symmetry $v_{z}^{*}(\rho, \bar{\rho})$ is not depended on angle $\varphi$ a given integral ca be considered in limit from 0 to $\pi / 2$. Then

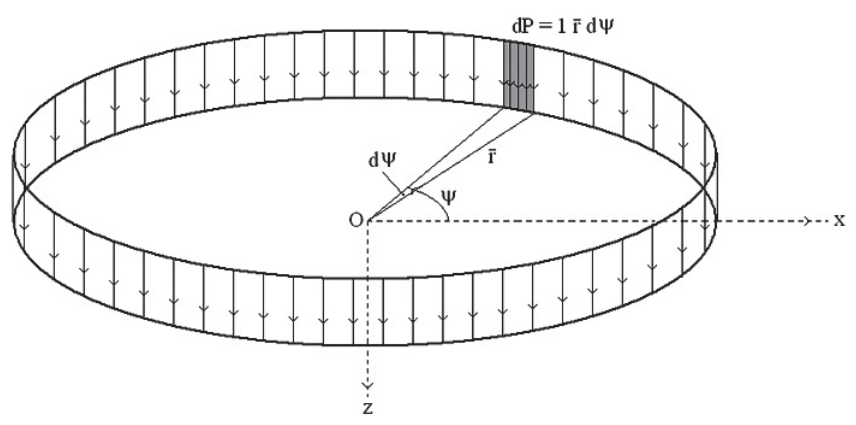

Fig. 3 Boundary of half-space loaded by unit uniformly loading on circular line of radius $\bar{r}$ $v_{z}^{*}(\rho, \bar{\rho})=\frac{4\left(1-\mu_{z}^{2}\right)^{\frac{\pi}{2}}}{\pi E_{z}} \int_{0}^{\frac{\bar{\rho} d \psi}{\sqrt{\rho^{2}+\bar{\rho}^{2}-2 \rho \bar{\rho} \cos \psi}} .}$

In solving the integral in (3), is necessary to separate the two cases: $\rho<\bar{\rho}$ and $\rho>\bar{\rho}$.

For the case $\rho<\bar{\rho}$, read

$v_{z}^{*}(\rho, \bar{\rho})=\frac{4\left(1-\mu_{z}^{2}\right)^{\frac{\pi}{2}}}{\pi E_{z}} \int_{0}^{\sqrt{1+\left(\frac{\rho}{\bar{\rho}}\right)^{2}-2\left(\frac{\rho}{\bar{\rho}}\right) \cos \psi}}$

and for the case $\rho>\bar{\rho}$, read

$v_{z}^{*}(\rho, \bar{\rho})=\frac{4\left(1-\mu_{z}^{2}\right)}{\pi E_{z}} \frac{\bar{\rho}^{\frac{\pi}{2}}}{\rho} \int_{0} \frac{d \psi}{\sqrt{1+\left(\frac{\bar{\rho}}{\rho}\right)^{2}-2\left(\frac{\bar{\rho}}{\rho}\right) \cos \psi}}$.

As it is shown (Rektorys 1963), integrals (4) and (5) are fully elliptic integrals of the first kind

$\int_{0}^{\frac{\pi}{2}} \frac{d \psi}{\sqrt{1+\left(\frac{\rho}{\bar{\rho}}\right)^{2}-2\left(\frac{\rho}{\bar{\rho}}\right) \cos \psi}}=\int_{0}^{\frac{\pi}{2}} \frac{d \psi}{\sqrt{1-\left(\frac{\rho}{\bar{\rho}}\right)^{2} \sin ^{2} \psi}}=\mathbf{K}\left(\frac{\rho}{\bar{\rho}}, \frac{\pi}{2}\right)$,

$\int_{0}^{\frac{\pi}{2}} \frac{d \psi}{\sqrt{1+\left(\frac{\bar{\rho}}{\rho}\right)^{2}-2\left(\frac{\bar{\rho}}{\rho}\right) \cos \psi}}=\int_{0}^{\frac{\pi}{2}} \frac{d \psi}{\sqrt{1-\left(\frac{\bar{\rho}}{\rho}\right)^{2} \sin ^{2} \psi}}=\mathbf{K}\left(\frac{\bar{\rho}}{\rho}, \frac{\pi}{2}\right)$.

Based on (6) and (7), the form for the displacements in (4) and (5) can be written more briefly:

for the case $\rho<\bar{\rho}$

$v_{z}^{*}(\rho, \bar{\rho})=\frac{4\left(1-\mu_{z}^{2}\right)}{\pi E_{z}} \boldsymbol{K}\left(\frac{\rho}{\bar{\rho}}, \frac{\pi}{2}\right)$

and for the case $\rho>\bar{\rho}$

$v_{z}^{*}(\rho, \bar{\rho})=\frac{4\left(1-\mu_{z}^{2}\right)}{\pi E_{z}} \frac{\bar{\rho}}{\rho} \boldsymbol{K}\left(\frac{\bar{\rho}}{\rho}, \frac{\pi}{2}\right)$.

Functions (8) and (9) represent the Green (influence) functions of the vertical displacement of the half-space boundary by unit loading, which is represented in Fig. 3, which allows for the determination of 
the displacements of the half space boundary by any axisymmetric segmental loading in the polar coordinates.

\subsection{Half-space circle loading}

Let us start with a study of the deformation surface boundary of the half-space, which is on the circle area of radius $R$ loaded by uniformly loading of permanent intensity $p_{1}=$ konst. (Fig. 1a).

The displacement function is necessary to construct for 2 areas: the area of loading $0 \leq r<R(0 \leq \rho<1)$ and the non loaded area $R \leq \rho$ $<\infty(1 \leq \rho<\infty)$ for the loading intensity $p_{1}$.

Based on (8) and (9), for the area $0 \leq \rho<1$ we have

$v_{z}(\rho)=\frac{4\left(1-\mu_{z}^{2}\right)}{\pi E_{z}}\left[\int_{0}^{\rho} \frac{\bar{\rho}}{\rho} \mathbf{K}\left(\frac{\bar{\rho}}{\rho}, \frac{\pi}{2}\right) p_{1} R d \bar{\rho}+\int_{\rho}^{1} \mathbf{K}\left(\frac{\rho}{\bar{\rho}}, \frac{\pi}{2}\right) p_{1} R d \bar{\rho}\right]$

and for the area $1 \leq \rho<\infty$

$v_{z}(\rho)=\frac{4\left(1-\mu_{z}^{2}\right)}{\pi E_{z}} \int_{0}^{1} \frac{\bar{\rho}}{\rho} \mathbf{K}\left(\frac{\bar{\rho}}{\rho}, \frac{\pi}{2}\right) p_{1} R d \bar{\rho}$.

Solving the integrals (10) and (11) by using the substitution

$\frac{\bar{\rho}}{\rho}=\xi \rightarrow \bar{\rho}=\rho \xi, \quad d \bar{\rho}=\rho d \xi$,

$\frac{\rho}{\bar{\rho}}=\eta \rightarrow \bar{\rho}=\rho \eta^{-1}, \quad d \bar{\rho}=-\frac{\rho}{\eta^{2}} d \eta$,

is given by Ryžik and Gradštein (Ryžik and Gradštein 1963, p. 641) in the following form:

for $0 \leq \rho \leq 1$

$$
\begin{aligned}
& v_{z}(\rho)=\frac{4\left(1-\mu_{z}^{2}\right) R p_{1}}{\pi E_{z}}\left\{\rho^{2}\left[\mathbf{E}\left(\xi, \frac{\pi}{2}\right)-\left(1-\xi^{2}\right) \mathbf{K}\left(\xi, \frac{\pi}{2}\right)\right]_{0}^{1}+\rho\left[\frac{\mathbf{E}\left(\eta, \frac{\pi}{2}\right)}{\eta}\right]_{1}^{\rho}\right\}= \\
& =\frac{4\left(1-\mu_{z}^{2}\right) R p_{1}}{\pi E_{z}} \mathbf{E}\left(\rho, \frac{\pi}{2}\right)
\end{aligned}
$$

and for $1 \leq \rho \leq \infty$

$$
\begin{aligned}
v_{z}(\rho) & =\frac{4\left(1-\mu_{z}^{2}\right) R p_{1}}{\pi E_{z}} \frac{1}{\rho}\left\{\rho^{2}\left[\mathbf{E}\left(\xi, \frac{\pi}{2}\right)-\left(1-\xi^{2}\right) \mathbf{K}\left(\xi, \frac{\pi}{2}\right)\right]_{0}^{\frac{1}{\rho}}\right\}= \\
& =\frac{4\left(1-\mu_{z}^{2}\right) R p_{1}}{\pi E_{z}} \rho\left[\mathbf{E}\left(\frac{1}{\rho}, \frac{\pi}{2}\right)-\left(1-\frac{1}{\rho^{2}}\right) \mathbf{K}\left(\frac{1}{\rho}, \frac{\pi}{2}\right)\right],
\end{aligned}
$$

where $\mathbf{K}\left(k, \frac{\pi}{2}\right)$ and $\mathbf{E}\left(k, \frac{\pi}{2}\right)$ are fully elliptical integrals of the first and second types given by

$\mathbf{K}\left(k, \frac{\pi}{2}\right)=\int_{0}^{\frac{\pi}{2}} \frac{d \omega}{\sqrt{1-k^{2} \sin ^{2} \omega}}=\frac{\pi}{2}\left\{1+\left(\frac{1}{2}\right)^{2} k^{2}+\left(\frac{1.3}{2.4}\right)^{2} k^{4}+\ldots+\left[\frac{(2 n-1) ! !}{2^{n} n !}\right]^{2} k^{2 n}+\ldots\right\}$,

$\mathbf{E}\left(k, \frac{\pi}{2}\right)=\int_{0}^{\frac{\pi}{2}} \sqrt{1-k^{2} \sin ^{2} \omega} d \omega=\frac{\pi}{2}\left\{1-\left(\frac{1}{2}\right)^{2} k^{2}-\left(\frac{1.3}{2.4}\right)^{2} \frac{k^{4}}{3}-\ldots-\left[\frac{(2 n-1) ! !}{2^{n} n !}\right] \frac{k^{2 n}}{2 n-1}-\ldots\right\}$ and $k=\rho, \frac{1}{\rho}$.

The deformation surface constructed using (13) and (14) is visible in Fig. 4. Some of itsdiscreet values are given in Tab. 1.

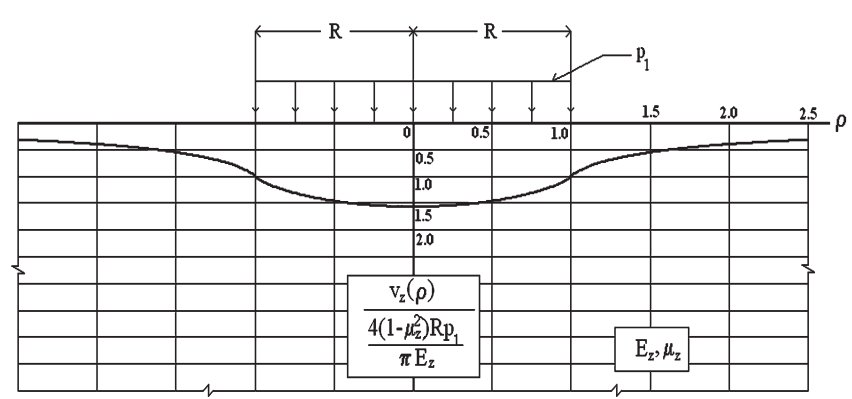

Fig. 4 Deformation surface boundary of the half-space caused by loading in Fig. 1 a

Tab. 1 Discreet values of the dimensionless displacements boundary of the half-space is visible in Fig. 4

\begin{tabular}{|c|c|c|c|c|c|c|c|}
\hline$\rho$ & $\mathbf{0}$ & $\mathbf{0 . 4}$ & $\mathbf{0 . 8}$ & $\mathbf{1 . 2}$ & $\mathbf{1 . 6}$ & $\mathbf{2 . 0}$ & $\mathbf{2 . 4}$ \\
\hline$\frac{v_{z}(\rho)}{4\left(1-\mu_{z}^{2}\right) R p_{1}}$ & & & & & & & \\
$\pi E_{z}$ & 1.5708 & 1.5059 & 1.2764 & 0.7365 & 0.5193 & 0.4063 & 0.3349 \\
\hline
\end{tabular}




\section{SIOYALY JOUSSILI \\ of

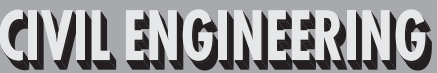

2010/2 PAGES $34-40$

\subsection{Half-space annular loading}

Now construct now the elastic deformation surface boundary of the half-space, which is on the annular area $r_{1} \leq r \leq r_{2}\left(\rho_{1} \leq \rho \leq \rho_{2}\right)$, which is loaded by the permanent loading of intensity $p_{2}=$ konst. (Fig. 1b).

It is necessary to construct the deformation function here for three areas. Based on (8) and (9):

for $0 \leq \rho<\rho_{1}$

$v_{z}(\rho)=\frac{4\left(1-\mu_{z}^{2}\right)}{\pi E_{z}} \int_{\rho_{1}}^{\rho_{2}} \mathbf{K}\left(\frac{\rho}{\bar{\rho}}, \frac{\pi}{2}\right) p_{2} R d \bar{\rho}$

for $\rho_{1} \leq \rho<\rho_{2}$

$v_{z}(\rho)=\frac{4\left(1-\mu_{z}^{2}\right)}{\pi E_{z}}\left[\int_{\rho_{1}}^{\rho} \frac{\bar{\rho}}{\rho} \mathbf{K}\left(\frac{\bar{\rho}}{\rho}, \frac{\pi}{2}\right) p_{2} R d \bar{\rho}+\int_{\rho}^{\rho_{2}} \mathbf{K}\left(\frac{\rho}{\bar{\rho}}, \frac{\pi}{2}\right) p_{2} R d \bar{\rho}\right]$,

for $\rho_{2} \leq \rho<\infty$

$v_{z}(\rho)=\frac{4\left(1-\mu_{z}^{2}\right)}{\pi E_{z}} \int_{\rho_{1}}^{\rho_{2}} \frac{\bar{\rho}}{\rho} \mathbf{K}\left(\frac{\bar{\rho}}{\rho}, \frac{\pi}{2}\right) p_{2} R d \bar{\rho}$,

where $\rho_{1}=\frac{R_{1}}{R}$ and $\rho_{2}=\frac{R_{2}}{R}$.

To solving integrals (16), (17) and (18), we can use substitutions

(12) and according to Ryžik and Gradštejn 1963, p. 641, we can write in closed form

for $0 \leq \rho<\rho_{1}$

$v_{z}(\rho)=-\frac{4\left(1-\mu_{z}^{2}\right) R p_{2}}{\pi E_{z}} \rho\left[-\frac{\mathbf{E}\left(\eta, \frac{\pi}{2}\right)}{\eta}\right]_{\frac{\rho}{\rho_{1}}}^{\frac{\rho}{\rho_{2}}}=$

$=\frac{4\left(1-\mu_{z}^{2}\right) R p_{2}}{\pi E_{z}}\left[\rho_{2} \mathbf{E}\left(\frac{\rho}{\rho_{2}}, \frac{\pi}{2}\right)-\rho_{1} \mathbf{E}\left(\frac{\rho}{\rho_{1}}, \frac{\pi}{2}\right)\right]$, for $\rho_{1} \leq \rho<\rho_{2}$

$$
\begin{aligned}
v_{z}(\rho) & =\frac{4\left(1-\mu_{z}^{2}\right) R p_{2}}{\pi E_{z}} \rho\left\{\left[\mathbf{E}\left(\xi, \frac{\pi}{2}\right)-\left(1-\xi^{2}\right) \mathbf{K}\left(\xi, \frac{\pi}{2}\right)\right]_{\frac{\rho_{1}}{\rho}}^{1}-\left[-\frac{\mathbf{E}\left(\eta, \frac{\pi}{2}\right)}{\eta}\right]_{1}^{\frac{\rho}{\rho_{2}}}\right\}= \\
& =\frac{4\left(1-\mu_{z}^{2}\right) R p_{2}}{\pi E_{z}}\left[-\rho \mathbf{E}\left(\frac{\rho_{1}}{\rho}, \frac{\pi}{2}\right)+\rho\left(1-\frac{\rho_{1}^{2}}{\rho^{2}}\right) \mathbf{K}\left(\frac{\rho_{1}}{\rho}, \frac{\pi}{2}\right)+\rho_{2} \mathbf{E}\left(\frac{\rho}{\rho_{2}}, \frac{\pi}{2}\right)\right]
\end{aligned}
$$

and for $\rho_{2} \leq \rho<\infty$

$$
\begin{aligned}
& \begin{aligned}
v_{z}(\rho) & =\frac{4\left(1-\mu_{z}^{2}\right) R p_{2}}{\pi E_{z}} \rho\left[\mathbf{E}\left(\xi, \frac{\pi}{2}\right)-\left(1-\xi^{2}\right) \mathbf{K}\left(\xi, \frac{\pi}{2}\right)\right]_{\frac{\rho_{1}}{\rho}}^{\frac{\rho_{2}}{\rho}}= \\
& =\frac{4\left(1-\mu_{z}^{2}\right) R p_{2}}{\pi E_{z}} \rho\left[\mathbf{E}\left(\frac{\rho_{2}}{\rho}, \frac{\pi}{2}\right)-\mathbf{E}\left(\frac{\rho_{1}}{\rho}, \frac{\pi}{2}\right)-\left(1-\frac{\rho_{2}^{2}}{\rho^{2}}\right)\right.
\end{aligned} \\
& \left.\mathbf{K}\left(\frac{\rho_{2}}{\rho}, \frac{\pi}{2}\right)+\left(1-\frac{\rho_{1}^{2}}{\rho^{2}}\right) \mathbf{K}\left(\frac{\rho_{1}}{\rho}, \frac{\pi}{2}\right)\right]
\end{aligned}
$$

We can easily prove, then that eqs. (19), (20) and (21) have the same values on their boundaries and represent a continuous deformation

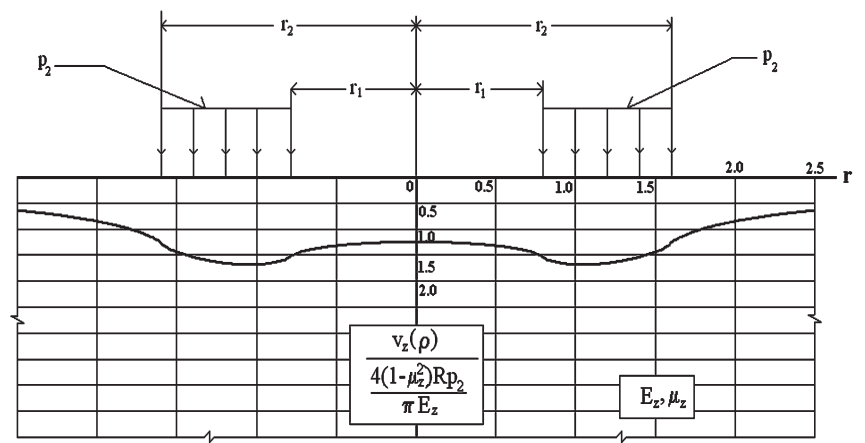

Fig. 5 Deformation of a boundary half-space, on the plane of the axis of symmetry $z$, caused by the loading imaged in Fig. $1 \mathrm{~b}$

Tab. 2 Discreet values of the dimensionless deformation boundary of the half-space represented in Fig. 5

\begin{tabular}{|c|c|c|c|c|c|c|c|}
\hline$\rho$ & $\mathbf{0}$ & $\mathbf{0 . 4}$ & $\mathbf{0 . 8}$ & $\mathbf{1 . 2}$ & $\mathbf{1 . 6}$ & $\mathbf{2 . 0}$ & $\mathbf{2 . 4}$ \\
\hline$\frac{v_{z}(\rho)}{4\left(1-\mu_{z}^{2}\right) R p_{2}}$ & & & & & & & \\
$\pi E_{z}$ & 1.2566 & 1.2992 & 1.5479 & 1.6594 & 1.2750 & 0.8594 & 0.6828 \\
\hline
\end{tabular}




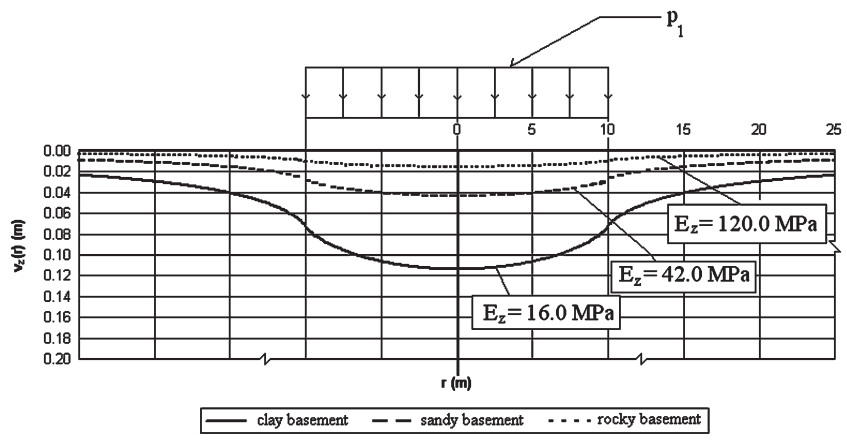

Fig. 6 Graph of the deformation of a half-space loaded by circular loading

surface. A graphic representation is given in Fig. 5 for $\rho_{1}=0.8$ and $\rho_{2}=1$.6. Tab. 2 provides some dimensionless values for axis $\rho$.

Other loading situations which are possible in practical applications can be easily constructed by a combination of the loading situations presented in Fig. 1a, b.

\section{PRACTICAL EXPERIMENTS}

\subsection{Half-space circle loading}

Inputs for circle loading:

$\mu_{\mathrm{z}}=0.3$,

$R=10 \mathrm{~m}$,

$p_{1}=p_{2}=0.1 \mathrm{MPa}$

The experiments for this loading case are presented for 3 different basements, for example: a clay basement $\left(E_{\mathrm{z}}=16.0 \mathrm{MPa}\right)$, a sandy basement $\left(E_{\mathrm{z}}=42.0 \mathrm{MPa}\right)$ and a rocky basement $\left(E_{\mathrm{z}}=120.0 \mathrm{MPa}\right)$. The deformation of a half-space by a permanent circle loading with a radius $R=10 \mathrm{~m}$ is given by Fig. 6 .

There is room for a question: How far from the structure is a measurable effect caused by the loading? In the case of the clayed basement a vertical displacement of magnitude $1 \mathrm{~cm}$ is approximately $57.1 \mathrm{~m}$, in the case of the sandy basement, approximately $22.3 \mathrm{~m}$, and in the case of the rocky basement, approximately $9.9 \mathrm{~m}$ from the centre of the loading. The vertical displacement of a magnitude of $1 \mathrm{~mm}$ is approximately $568 \mathrm{~m}$ in the case of the clayed basement, in the case of the sandy basement, approximately $217 \mathrm{~m}$, and in the case of the rocky basement, approximately $76.4 \mathrm{~m}$ from the centre of the loading.

\subsection{Half-space annular loading}

The loading of a half-space by annular loading with radii of $r_{1}=8 \mathrm{~m}$

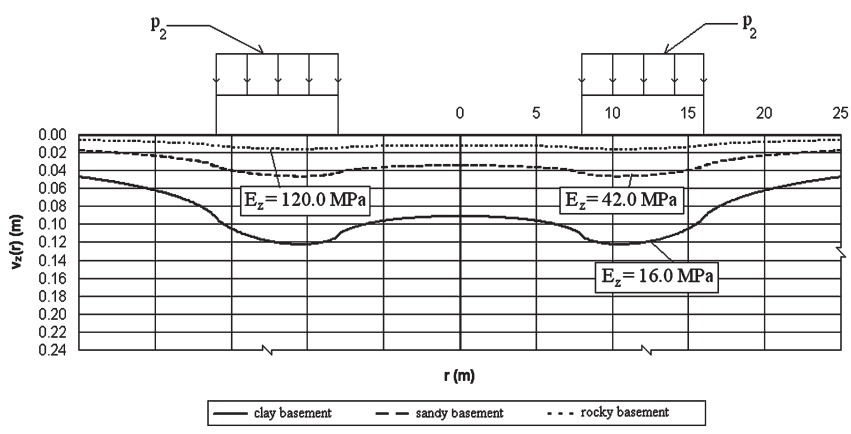

Fig. 7 Graph of a deformation surface with annular loading

and $r_{2}=16 \mathrm{~m}$ with the same physical inputs as in sec. 3.1 , is imaged in Fig. 7.

In this case of deformation, the vertical displacement of a magnitude of $1 \mathrm{~cm}$ is in case of the clayed basement, approximately $110 \mathrm{~m}$, in the case of the sandy basement, approximately $42 \mathrm{~m}$ and in the case of the rocky basement, approximately $18 \mathrm{~m}$ from 'the centre of the loading. The vertical displacement of magnitude of $1 \mathrm{~mm}$ is, in the case of the clayed basement, approximately $1010 \mathrm{~m}$, in the case of the sandy basement, approximately $416 \mathrm{~m}$, and in the case of the rocky basement, approximately $148 \mathrm{~m}$ from the centre of the loading.

\section{CONCLUSION}

This study provides basic information about the deformation of the Earth's surface by circular or annular loading caused by motion from the accumulation of a mass (tall buildings, large dams, filling or cutting near highways, the accumulation of a water mass, the accumulation of a snow mass, etc.). The modelling of loading effects is very useful in geodesy. A suitable solution means eliminating time-dependent systematic error and increasing the accuracy of geodetic networks. It can be used in the construction and realisation of geodetic reference networks, the analysis of repeat measurements, building stability, etc. It is necessary to undertake take many geodetic experiments in order to prove theoretical models or for improve the mathematical - physical models of loading.

\section{ACKNOWLEDGEMENT}

This project was supported by Grant Agency of the Slovak Republic (1/0882/08) and by Grant Agency for Science and Research of the Slovak Republic (LPP-0216-06). 


\section{IOVALS JOUSSIAL \\ O)

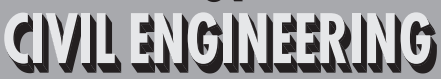

2010/2 PAGES $34-40$

\section{REFERENCES}

[1] Rektorys, K. (1963): Přehled užité matematiky. Státni nakladatelství technické literatúry, Praha, in English: Review of Applied Mathematics.

[2] Ryžik, J.M., Gradštejn, J.S. (1963): Tablicy integralov, summ rjadov i proizvedenii. Gosud. izdat. fiz-mat. lit., Moskva, in English: Tables of Integrals, Sum of Series and Products.
[3] Boussinesq, J. (1885): Applications des potentials ŕ létude de léquilibre et du mouvement des solides élastiques. Paris, Gauthier - Villars 1885, 558s. 